\title{
Epithelial Cell Segmentation in Histological Images of Testicular Tissue Using Graph-Cut
}

\author{
Azadeh Fakhrzadeh ${ }^{1}$, Ellinor Spörndly-Nees ${ }^{2}$, Lena Holm ${ }^{2}$, \\ and Cris L. Luengo Hendriks ${ }^{1}$ \\ 1 Centre for Image Analysis, \\ ${ }^{2}$ Department of Anatomy, Physiology and Biochemistry, \\ Swedish University of Agricultural Sciences, Uppsala, Sweden \\ \{azadeh. fakhrzadeh, cris\}@cb.uu.se, \\ \{Ellinor.Sporndly-Nees, Lena.Holm\} @slu.se
}

\begin{abstract}
Computerized image processing has provided us with valuable tools for analyzing histology images. However, histology images are complex, and the algorithm which is developed for a data set may not work for a new and unseen data set. The preparation procedure of the tissue before imaging can significantly affect the resulting image. Even for the same staining method, factors like delayed fixation may alter the image quality. In this paper we face the challenging problem of designing a method that works on data sets with strongly varying quality. In environmental research, due to the distance between the site where the wild animals are caught and the laboratory, there is always a delay in fixation. Here we suggest a segmentation method based on the structural information of epithelium cell layer in testicular tissue. The cell nuclei are detected using the fast radial symmetry filter. A graph is constructed on top of the epithelial cells. Graph-cut optimization method is used to cut the links between cells of different tubules. The algorithm is tested on five different groups of animals. Group one is fixed immediately, three groups were left at room temperature for 18, 30 and 42 hours respectively, before fixation. Group five was frozen after 6 hours in room temperature and thawed. The suggested algorithm gives promising results for the whole data set.
\end{abstract}

\section{Introduction}

The pathologist analyses tissue slides using light microscopy to detect morphological abnormalities. Statistical quantification of histological aberrations requires evaluation of a large number of slides, which is time consuming. Manual analysis is subjective and there is always a risk that pathologists vary in the assessment of a tissue due to tedious repetition of the work. The histological slides can be digitized, which enables the use of computerized image analysis and machine learning techniques to complement the evaluation of the pathologist. Such computer-assisted diagnosis is receiving the attention of many researchers.

Several reports indicate that compounds found in the environment can affect male reproduction in humans, mammals, birds and fish [2]. Histopathology of testicular tissue is regarded as a sensitive tool for detecting adverse effects caused by chemicals in male 
reproduction [8]. One of the first steps in computerised analysis of histological images is to identify different structures, like cells, lumen, interstitial tissue and tubules. Once these structures are segmented, measurements of different features can be carried out to detect adverse morphology. Segmentation will be done by using chromatic information or spatial relationship of different components. The final image quality may vary because of the preparation procedure. It is a challenging problem to propose a method that works correctly, with the same parameters, on any data set, even for the same tissue and same staining method. To complicate matters more, prolonged time between the death of the animal and fixation of testis affects the histology [5]. This is relevant in environmental research due to the distance between the site were the wild animals are caught and the laboratory. For practical reasons the animal may even be frozen. This generates evaluation difficulties due to autolysis and freeze damages of the tissue. The chromatic information may also be affected by the delayed fixation and freezing.

In this paper we suggest a segmentation method that uses the structural information of the testicular epithelium marked with GATA-4 antibody. This method can be used on any tissue with similar structure. The spatial relationship of the cells has been used for epithelium classification [9]15|6|10|13]. Albert et al. use a graph theoretical method to study the morphological characteristics of the epithelium [1]. The minimal spanning tree is computed in the three-dimensional (3D) space of the sections with the selected centers of the nuclei as vertices. The average length of all edges in the graph is used for discriminating different specimens. Bilgin et al. [3] first use k-means clustering to segment the epithelium and then the cell graph is used for classifying brain tissue samples. Gunduz et al. [9] also construct a graph on top of the cells and then compute the graph metrics of the cell graphs, including the degree, clustering coefficient, eccentricity and closeness for each cell to distinguish healthy from unhealthy tissue. Here we also use graphs, but as a segmentation method.

\section{Methodology}

The proposed algorithm first segments the cell nuclei, which form the vertices of a graph. A graph $g(\nu, \varepsilon)$ is defined as a set of nodes or vertices $\nu$ and a set of edges $\varepsilon$, connecting neighbouring nodes. The graph-cut optimisation is used to remove the links between nuclei belonging to different tubules, which thus yields an isolated sub-graph for each tubule. By applying morphological operators on sub-graphs we can delineate the outer boundary of the epithelium.

\subsection{Cell Segmentation and Vertices Identification}

The cell nuclei are mostly radially symmetric, several methods are suggested to measure the local symmetry in the image. Kuse et al. [12] used phase symmetry suggested by Kovesi [11] for segmenting cells, we use the fast radial symmetry filter here to extract them [13]. If pixel $p$ is located on the arc of a circle then the center of the circle is at one radius distance in the direction of the gradient. The Fast radial symmetry filter is calculated at one or more radii. Loy et al. [13] first calculate the Magnitude projection 
image $M_{n}$ and orientation projection image $O_{n}$ which have high response at potential centroids. $M_{n}$ and $O_{n}$ in [13] are defined as:

$$
\begin{gathered}
M_{n}\left(P_{ \pm v e}(p)\right)=M_{n}\left(P_{ \pm v e}\right) \pm\|\vec{g}(p)\|, \\
O_{n}\left(P_{ \pm v e}(p)\right)=O_{n}\left(P_{ \pm v e}\right) \pm 1,
\end{gathered}
$$

where $\vec{g}(p)$ is the gradient at pixel $p$ and $P_{ \pm v e}$ is a "positively/negatively affected pixel" and is calculated using the gradient:

$$
P_{ \pm v e}=P \pm \operatorname{round}\left(\frac{\vec{g}(p)}{\|\vec{g}(p)\|} n\right) .
$$

The radial symmetry contribution at radius $n$ is

$$
S_{n}=\tilde{M}_{n}(p)\left|\tilde{O}_{n}(p)\right|^{\alpha} \otimes A_{n}
$$

where $\alpha$ is a scaling factor, $A_{n}$ is a two-dimensional Gaussian with the standard deviation proportional to n, $\tilde{M}_{n}$ and $\tilde{O}_{n}$ are normalized $M_{n}$ and $O_{n}$ across different radii, and $\otimes$ denotes the convolution. We set the value of $\alpha$ equal to one and the standard deviation of $A_{n}$ to $n / 4$. The result of the filter is the average of the radial symmetry over different values of $n$. Segmentation of the radial symmetry yields the epithelial cell nuclei, but also other nuclei. We formed a feature vector based on the morphology of the cells (size, perimeter) and statistical intensity information (mean, standard deviation, skewness, minimum value and maximum value of the lightness channel of $L^{*} a^{*} b^{*}$ color space), then applied k-means clustering to cluster cells into two groups. The epithelial cells which have lower mean value are used for further steps.

\subsection{Edge Establishment}

In order to establish the edges $\varepsilon$ of our graph we need to find the neighbouring cell nuclei centroids. One way is to use the Delaunay triangulation. The Delaunay triangulation of a set of $m$ points corresponds to the dual graph of its Voronoi diagram. The Voronoi diagram divides the space into $m$ polygons $\left\{P_{1}, P_{2}, \ldots P_{m}\right\}$, where $P_{a}$ corresponds to the point $S_{a}$. A point $c$ belongs to polygon $P_{a}$ if

$$
d\left(c, S^{a}\right)=\min _{j} d\left(c, S_{j}\right),
$$

where $j \in\{1,2, . . m\}$ and $d\left(c, S_{j}\right)$ is the Euclidean distance between $c$ and $S_{j}$. Two points $S_{a}$ and $S_{b}$ share an edge in the Delaunay triangulation if their corresponding polygons $P_{a}$ and $P_{b}$ share a side in the Voronoi diagram. Delaunay triangulation yields a graph with edges only between adjacent vertices. A sample of such a graph is shown in Figure 10 To make the graph simpler we removed the edges that are larger than a threshold. This threshold is proportional to the largest distance between neighboring cells inside the tubules, which is 100 pixels for this application. 


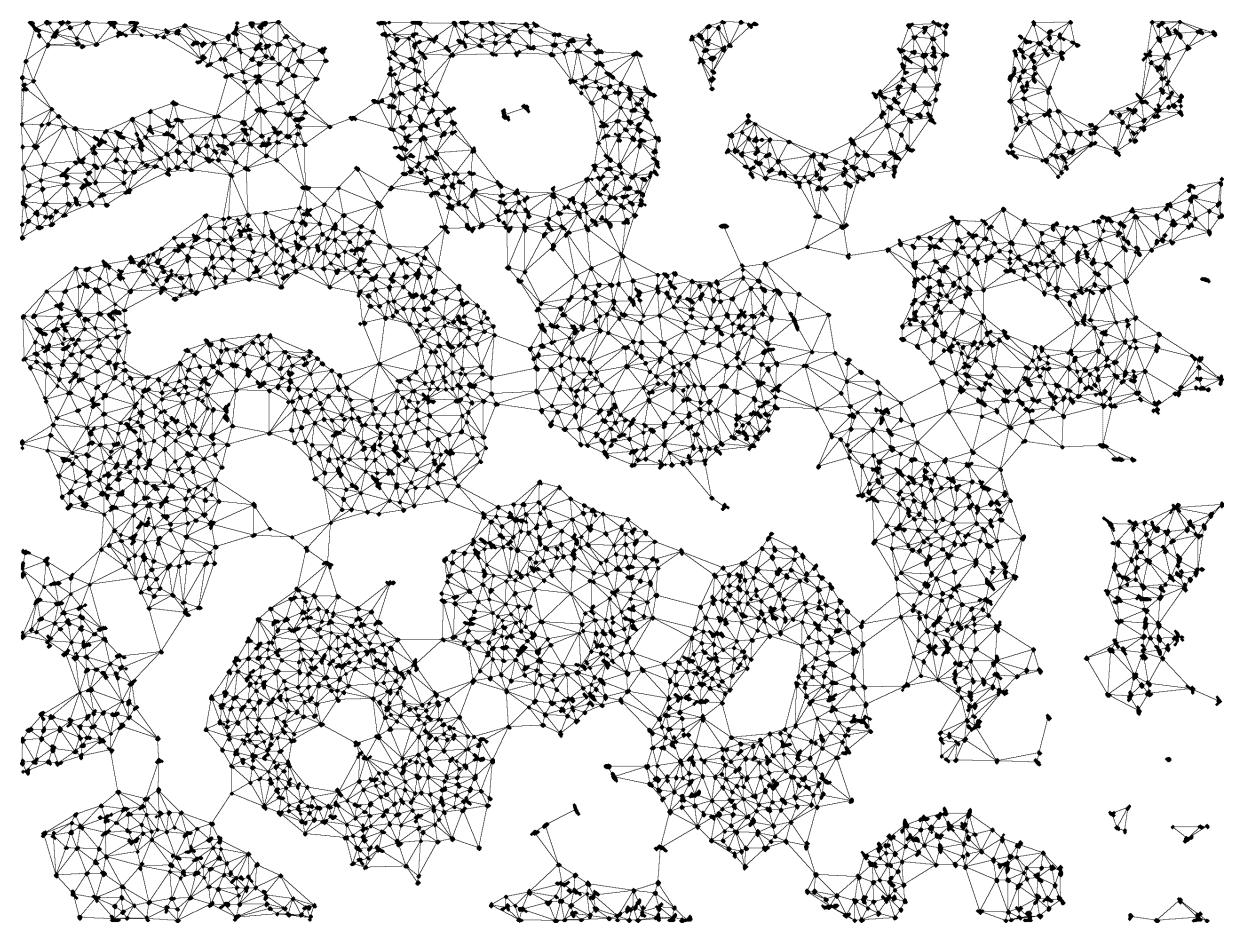

Fig. 1. A graph constructed on top of Gata-4 marked germ cells

\subsection{Edge Weights}

As you can see in Figure 1 the Gata-4 marked germ cells usually form a cluster around a center. The distance between two nuclei in the same tubule is smaller than the distance between nuclei in different tubules. We set the weight of each edge to be inversely proportional to the Euclidean distance between the two vertices that it connects.

\subsection{Graph-Cut Minimization}

We use a graph-cut minimization method to remove undesired edges. In graph-cut method we need to specify two special terminal nodes which are called $\mathrm{S}$ (source) and $\mathrm{T}$ (sink) that represent object and background labels. Edges between vertices are called nlinks, and t-links represent connections between vertices and terminals. All graph edges $e \in \varepsilon$ including t-links and n-links are assigned some non-negative cost $w_{e}$. An s-t cut is a subset of edges $C \subset \varepsilon$ such that the terminals $\mathrm{S}$ and $\mathrm{T}$ become completely separated on the induced graph. The cost of cut is sum of the weights of all the edges it severs which is :

$$
|C|=\sum_{e \in C} w_{e}
$$


A minimum s-t cut, is a cut with minimal cost. Based on combinational optimization, a globally minimum s-t cut can be computed efficiently in low order polynomial time. Boykov et al. [4] introduced a new version of the max-flow algorithm that outperformed existing techniques for computer vision applications. We need interaction to specify the source and the sinks of the graph.

\subsection{Animals and Tissue Preparation}

Twenty five healthy, sexually mature minks were collected at the annual culling on a mink farm. The animals were divided into five groups based on time interval between euthanization and fixation. Group one was put in fixative immediately post mortem. Three groups were left at room temperature for 18, 30 and 42 hours respectively, before fixation. Group five was frozen after 6 hours in room temperature and thawed (6h+frozen). Transverse tissue slices from testis were fixed in modified Davidsons fluid for 24 hours at $4^{\circ} \mathrm{C}$ and embedded in paraffin wax. The samples were cut in 5 micrometer sections and stained with GATA-4 antibody. Digital images of the sections were taken with a Nikon Microphot-FXA microscope using a 10x objective lens.

\section{Results and Discussion}

The testicular tissue is composed of seminferous tubules and interstitial tissue. Chemicals or delayed fixation can alter the morphology of cells in the tubules. The quality of images from mink testis fixed immediately (zero hour) differs from the those fixed after 30 hours, (Figures 2a and 2d), but the overall structure is conserved regardless of storage time. We applied the method outlined earlier to segment the tissue sections into individual tubules. To improve the result, we first applied the bilateral filter. We chose a spatial-domain standard deviation of 3 pixels and an intensity domain standard deviation of 0.2 times the dynamic range of the image. We then applied the fast radial symmetry filter with radii 3,4 and 5 pixels, which are suitable radii for the size of the cells in the images. The thresholded result gave the nuclei. As we can see in the sample images in Figure 2] there were two different cell types in the tissue. We clustered the nuclei in two groups using k-means clustering. The feature vector used contained the mean, standard deviation, skewness, minimum value and maximum value of the lightness channel of $L^{*} a^{*} b^{*}$ color space, and the size and perimeter of the nulei. Next we applied Delaunay triangulation to the epithelial nuclei to create a graph. The weight of edges were inversely proportional to the Euclidean distance between two vertices that they connected. The user was asked to add markers at the center of each tubule to be separated. The markers were grown until they each hit 7 nuclei. These nuclei were taken as sources and sinks of the graph. The max-flow optimisation was run and the links between different tubules were removed.

Figure 2c and 2d shows the graphs, overlaid on top of the images. The edges shown in red are those which were cut. The graphs associated to every tubule can be used directly for classification, or we can use mathematical morphological operators to obtain the whole area enclosed by the graph and apply more traditional image-based features 


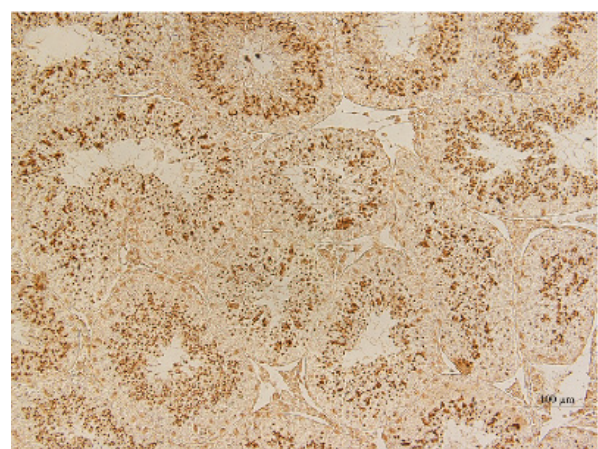

(a)

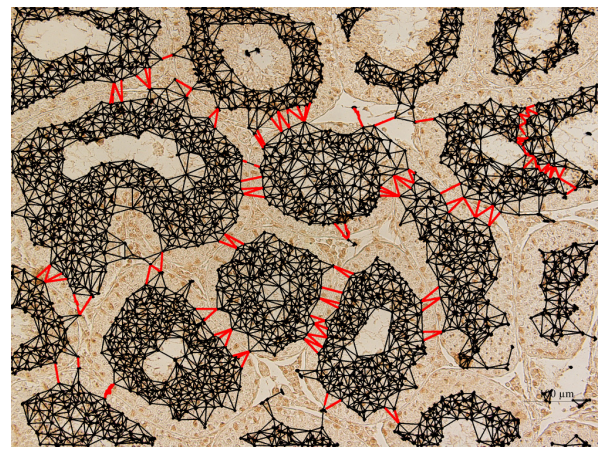

(c)

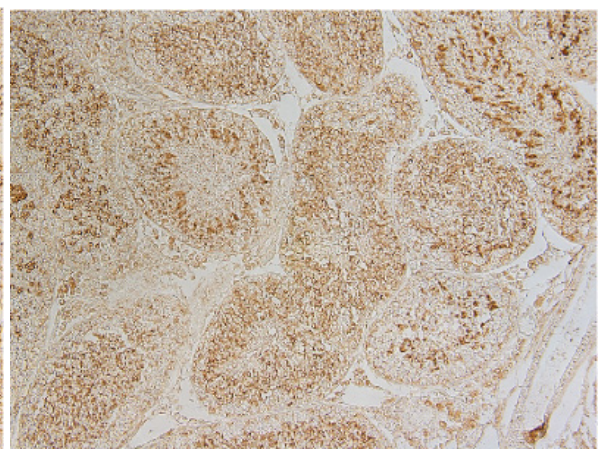

(b)

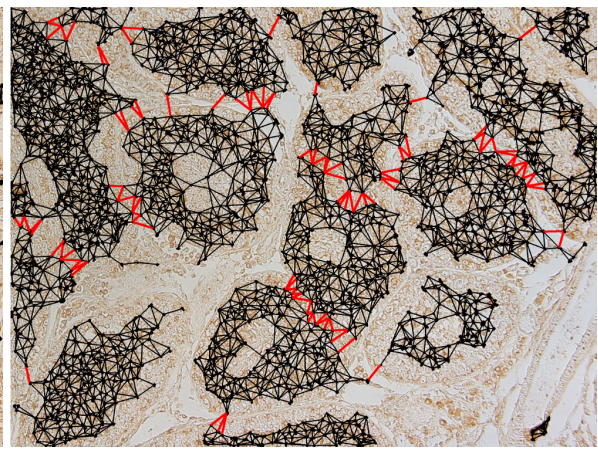

(d)

Fig. 2. (a) Stained thin section of mink testicular tissue, fixated immediately after euthanasia. (b) Stained thin section of mink testicular tissue, fixated 30 hours postmortem. (c) and (d) Graphs constructed with epithelial nuclei as vertices, for the images in (a) and (b), respectively. Red edges are removed by the proposed method to separate the various tubules.

for classification. We applied our method on 50 images, 10 images per group. A subgraph associated to a tubule which excludes some of the epithelial cells of the tubule, and a sub-graph which includes some of the cells from neighboring tubules both are considered as wrongly segmented. Similarly, a small cell cluster that does not belong to any tubule is a wrong segmentation. For group 1 (zero hour postmortem) $85 \%$ of epithelial cell layer of tubules were segmented correctly. For groups 2, 3, 4 and 5, this number is $66 \%, 71 \%, 76 \%$, and $72 \%$ respectively. The number of tubules per group are, $166,158,129,138,124$. As was expected for the zero hour, in which the structure of the tissue is preserved better, the error is smaller than for the other groups.

\section{Conclusion}

A semi-automated method for segmenting the epithelial cell layer was proposed. The proposed method uses the structural information of the tissue and can be used on im- 
ages of every tissue with similar structure. Initially, the cell nuclei of the epithelium is delineated. These cell nuclei serve as nodes of graph. The neighboring nuclei are connected by graph edges and the weight of the edges is inversely proportional to the distance between nodes. The method was tested on imaged tissue with up to 42 hours delay in fixation, or frozen prior to fixation which yielded promising results even though the quality of images vary a lot.

The result of segmentation can be used to analyse the structure of the seminiferous epithelium, establish postmotem effect of delayed fixation and to find endpoints to detect adverse effects. When analysing material from wild animals, it is important to separate the histological changes caused by delayed fixation or freezing postmortem from premortem pathology.

Acknowledgment. Authors of this paper would like to acknowledge Dr. Elisabeth Ekstedt, Prof. Gunilla Borgefors and Rahim KadkhodaMohammadi for the helpful discussions.

\section{References}

1. Albert, R., Schindewolf, T., Baumann, I., Harms, H.: Three dimensional image processing for morphometric analysis of epithelium sections. Cytometry 13, 1-18 (1992)

2. Bergman, A., Heindel, J.J., Jopling, S., Kidd, K.A., Zoeller, R.T.: State of the science of endocrine disrupting chemicals 2012: an assessment of the state of the science of endocrine disruptors prepared by a group of experts for the United Nations Environment Programme and World Health Organization. World Health Organization, Geneva (2013)

3. Bilgin, C., Demir, C., Nagi, C., Yener, B.: Cell-graph mining for breast tissue modeling and classification. In: 29th Annu. Int. Conf. IEEE EMBS, vol. 13 (2007)

4. Boykov, Y., Kolmogorov, V.: An Experimental Comparison of Min-Cut/Max-Flow Algorithms for Energy Minimization in Vision. IEEE Transactions on Pattern Analysis and Machine Intelligence 26, 1124-1137 (2004)

5. Bryant, B.H., Boekelheide, K.: Time-dependent changes in post-mortem testis histopathology in the rat. Birth Defects Res. B Dev. Reprod. Toxicol. 35, 665-671 (2007)

6. Choi, H.K., Jarkrans, T., Bengtsson, E., Vasko, J., Wester, K., Malmstrom, P.U., Busch, C.: Image analysis based grading of bladder carcinoma. Comparison of object, texture and graph based methods and their reproducibility. Anal. Cell Pathol. 15, 1-18 (1997)

7. Clarke, F., Ekeland, I.: Nonlinear oscillations and boundary-value problems for Hamiltonian systems. Arch. Rat. Mech. Anal. 78, 315-333 (1982)

8. Creasy, D.M.: Evaluation of testicular toxicology: a synopsis and discussion of the recommendations proposed by the Society of Toxicologic Pathology. Birth Defects Res. B Dev. Reprod. Toxicol. 68, 366-367 (2003)

9. Gunduz, C., Yener, B., Gultekin, S.H.: The cell-graphs of cancer. In: 12th International Conference on Intelligent Systems for Molecular Biology (ISMB), Glasgow, Scotland (August 2004)

10. Keenan, S.J., Diamond, J., McCluggage, W.G., Bharucha, H., Thompson, D., Bartels, B.H., Hamilton, P.W.: An automated machine vision system for the histological grading of cervical intraepithelial neoplasia (CIN). J. Pathol. 192, 351-362 (2000)

11. Kovesi, P.: Local isotropic phase symmetry measure for detection of beta cells and lymphocytes. In: 10th Australian Joint Conf. Artificial Intelligence, pp. 15-20 (1997) 
12. Kuse, M., Wang, Y.F., Kalasannavar, V., Khan, M., Rajpoot, N.: Local isotropic phase symmetry measure for detection of beta cells and lymphocytes. J. Pathol. Inform. 2(2) (2011)

13. Loy, G., Zelinsky, A.: A Fast Radial Symmetry Transform for Detecting Points of Interest. In: Heyden, A., Sparr, G., Nielsen, M., Johansen, P. (eds.) ECCV 2002, Part I. LNCS, vol. 2350, pp. 358-368. Springer, Heidelberg (2002)

14. Naik, S., Doyle, S., Feldman, M., Tomaszewski, J., Madabhushi, A.: Gland segmentation and computerized Gleason grading of prostate histology by integrating low-high-level and domain specific information. In: Proceedings of MIAAB (2007)

15. Weyn, B., Van de Wouwer, G., Kumar-Singh, S., Van Daele, A., Scheunders, A., Van Marck, E., Jacob, W.: Computer-assisted differential diagnosis of malignant mesothelioma based on syntactic structure analysis. Cytometry 35, 23-29 (1999) 